\title{
Lumbar Rib
}

National Cancer Institute

\section{Source}

National Cancer Institute. Lumbar Rib. NCI Thesaurus. Code C158330.

Presence of rib formation in the lumbar region. 